\title{
A possible model for initiation of ULF oscillation in magma
}

\author{
Yuri A. Kopytenko and Lidia V. Nikitina \\ St. Petersburg Department of Institute of Terrestrial Magnetism, Ionosphere and Radiowave \\ Propagation (SPbFIZMIRAN), Russian Adademy of Science, St. Petersburg, Russia
}

\begin{abstract}
During the period just prior to an earthquake, an electomagnetic emission develops over seismic zones. In this paper, a model of excitation of magnetic fields over zones of volcanic activity is proposed. Movement of magma along volcanic channels precedes an earthquake, hydrodynamic processes in the moving magma can lead to formation of waves and vortices in the flow which, in turn, can cause development of magnetic fields in conducting magma. During this period, the movement of the magma intensifies leading to a corresponding intensification of the magnetic fields. In this paper, different possible sources of ULF pulsation in magma are examined, and the variable geomagnetic fields induced by this pulsation are estimated.
\end{abstract}

Key words ULF oscillations - magma - volcano - electromagnetic emission

\section{Introduction}

Seimoelectric and seisomomagnetic effects in rock were first measured and described in the late 1930's, and since that time have been the subject of various analytical investigations. The electromagnetic effects are explained by tectonic processes that occur in rock prior to an earthquake (see, e.g., Stacey, 1964; Mogi, 1985). In this article, another possible model of electromagnetic excitation over an active volcanic zone is proposed.

Consider magma as a two-phase liquid (liquid and gas) with viscosity of approximately $v=5$ $\mathrm{m}^{2} / \mathrm{s}$ and a density $\rho \approx 2500 \mathrm{~kg} / \mathrm{m}^{3}$ (Marchinin, 1985). Initially, magma is found at a depth of ap-

Mailing address: Dr. Lidia V. Nikitina, St. Petersburg Department of Institute of Terrestrial Magnetism, Ionosphere and Radiowave Propagation (SPbFIZMIRAN), Russian Adademy of Science, p/b 188, Muchnoi 2, 191023 St. Petersburg, Russia; e-mail: lida@mail.spbnit.ru proximately $100 \mathrm{~km}$. When volcanic activity increases, magma moves upward toward the Earth's surface through volcanic channels, filling intermediate volcano capacities whose depth can vary from 2 to $20 \mathrm{~km}$ and whose radii vary. For example, the capacity under the Kluchevskii volcano is estimated to be $4 \mathrm{~km}$ deep with a radius of 3600 $\mathrm{m}$ (Fedotov et al., 2000) and the capacity under the Shiveluch volcano is $20 \mathrm{~km}$ deep with a radius of $6000 \mathrm{~m}$. Just prior to an earthquake, the movement of magma becomes more intense, and waves and vortices develop. In the presence of the Earth's constant magnetic field, these movements induce the formation of variable magnetic fields with different periods which then contribute to the common electromagnetic emission that is present before the earthquake starts (Ismaguilov et al., 2001.) We will now discuss the possible mechanisms of excitation of magnetic fields in magma and estimate their magnitude.

\section{Hydrodynamic processes in magma}

Consider three kinds of hydrodynamic processes in magma that can lead to electromagnetic emission. 


\subsection{Lifting of magma in magma channels}

Prior to the seismic event, magma moves in volcanic channels toward the ground's surface. Magma velocity is approximately $1-5 \mathrm{~m} / \mathrm{s}$ (Marchinin, 1985; Slezin, 1999). The electromagnetic fields can be induced by magma flow in the presence of the Earth's constant magnetic field. These fields are quasi-constant, and their values depend on the velocity of rising magma and the radius of the volcanic channel.

\subsection{Oscillations at the magma surface}

The possible sources of the ULF electromagnetic pulsations are the oscillations that can appear at the magma surface (fig. 1). Inertial waves appear on the surface of liquid between two media with different densities. The frequency of waves on the magma surface depends on the relative change of density between the gas and the magma and on the diameter of the channel.

$$
f^{2}=\left(\left(\rho_{2}-\rho_{1}\right) /\left(\rho_{2}+\rho_{1}\right)\right) g k
$$

where $k$ is the wave number, $\rho_{1}$ is the gas density, $\rho_{2}$ is the magma density. Relative changes in the density can be estimated as $\left(\rho_{2}-\rho_{1}\right) /\left(\rho_{2}+\right.$ $\left.+\rho_{1}\right) \approx 0.5$.

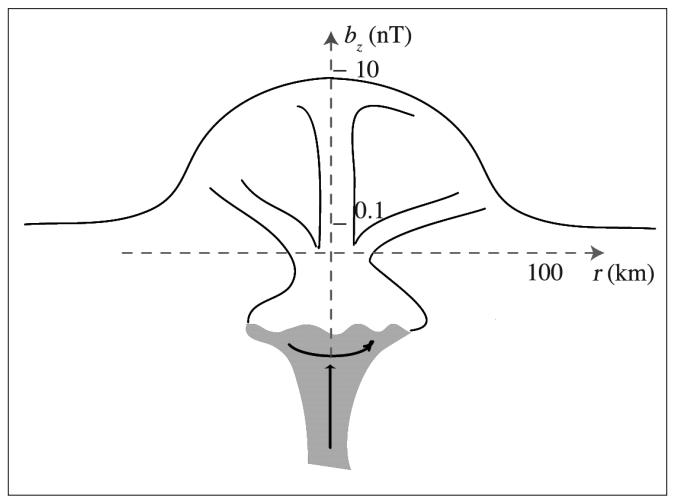

Fig. 1. Oscillations and waves in a volcano capacity. The magnetic field $b_{z}$ induced by the vortex in the volcano capacity.
If the magma surface is in the intermediate volcanic capacity with diameter $d \approx 10^{3} \mathrm{~m}(k=$ $\left.=10^{-3} \mathrm{~m}^{-1}\right)$ then according to (2.1) oscillations exciting in magma have frequencies of approximately $f=10^{-2} \mathrm{~Hz}(T=100 \mathrm{~s})$. If magma is in a channel with a diameter of approximately 100 $\mathrm{m}$ then oscillations with period ranging 10-30 s are excited in the magma. Bubbles of gas disturb the magma surface constantly and support the oscillations on this surface.

Increasing volcanic activity leads to the appearance of new gas bubbles. Gas moves in a channel with a velocity greater than the upwelling velocity of the magma (Proussevitch et al., 1993; Slezin, 1999). Step by step, a large volume of bubbles accumulates at the magma surface where they create a foam structure. This leads to a smaller difference in density between the magma and the gas, and inertial waves become weaker. The decrease in wave intensity must be typical for the duration of the preearthquake period when a large amount of gas accumulates in the magma.

After the magma becomes free of the «foam cap» (for example, when new cracks appear), the density difference between the gas and the magma is restored, and inertial waves are initiated at the magma surface again. So, at the frequency of inertial waves we can observe that an increase in ULF magnetic pulsation is replaced by a decrease in oscillation corresponding to increased volcanic activity.

\subsection{Influence of Coriolis force to the magma movement}

Another cause of the ULF movements in magma is related to vortex structures that appear in magma flow (see fig. 1). When magma is filling a large intermediate volcano capacity, vortex flow appears in magma according to Coriolis force

$$
v_{\varphi}^{2} / R=2 \Omega v_{r}
$$

where $R$ is the vortex radius, $\Omega$ is the angle velocity of the Earth's rotation, $\Omega \approx 7 \cdot 10^{-5} c^{-1}, v_{\varphi}$ is the rotation velocity of liquid, $v_{r}$ is the radial velocity. If we assume that $v_{r}$ is approximately 
equal to the upwelling velocity of magma in the channel and the radius of the volcanic center is approximately $R=1000 \mathrm{~m}$, then the liquid rotates with angle velocity $5.10^{-3} \mathrm{rad} / \mathrm{s}$ and rotation velocity $v_{\varphi}=5 \mathrm{~m} / \mathrm{s}$.

\section{Electromagnetic emission induced by hydrodynamic processes in magma}

Let us estimate the magnetic fields induced by the processes in magma described above. The most difficult problem connected with the estimation of electromagnetic fields is estimating the conductivity of magma. Conductivity of basalts is estimated to be $10^{-3} \mathrm{Sm} / \mathrm{m}$ at the Earth's surface, but their conductivity depends strongly on temperature. Magma temperature in volcano channels reaches $1000-1300^{\circ} \mathrm{C}$. If temperature increases to the values $500-1000^{\circ}$ then conductivity increases on 2-3 fold (Van'yan, 1983). According to these data we assume that conductivity of hot magma is approximately $\sigma=10^{-1} \mathrm{Sm} \cdot \mathrm{m}^{-1}$.

\subsection{Magnetic fields induced by rising magma flow}

Assume lifting magma in a channel is similar to the cylindrical flow of a conducting liquid. The magnetic field induced by the cylindrical flow can be estimated as (Parker, 1979)

$$
B_{Z} \approx \mu \sigma B_{0 x} R v_{Z}
$$

where $B_{0 x}$ is the horizontal component of the Earth's magnetic field, $R$ is the radius of the cylinder. If we assume that $\sigma=10^{-1} \mathrm{Sm} \cdot \mathrm{m}^{-1}, B_{0 x}=$ $=2 \cdot 10^{4} \mathrm{nT}, v_{z}=1-5 \mathrm{~m} / \mathrm{s}$ and the capacity radius is $R=1000 \mathrm{~m}$ then $B_{z} \approx 2-10 \mathrm{nT}$ (fig. 2 ). The increase in the magnetic field during early seismic activity can be initiated by the acceleration of the flow.

\subsection{Electromagnetic emission induced by inertial oscillations}

The vertical component of the magnetic field $b_{z}$ induced by inertial oscillations can be

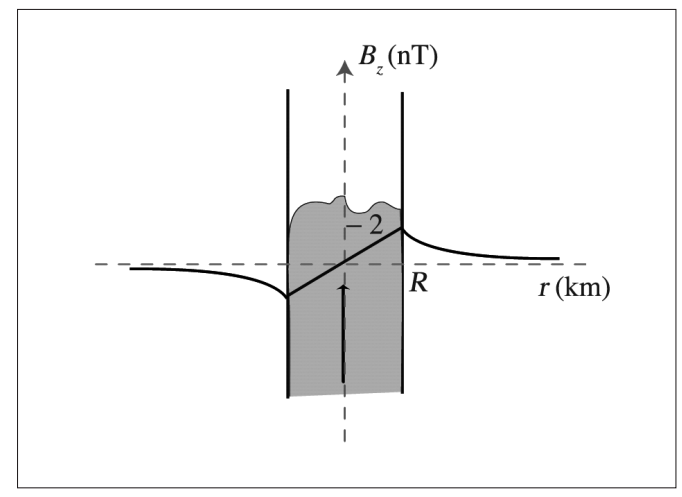

Fig. 2. The magnetic field $B_{z}$ induced by the lifting flow of magma. $R$ is the radius of the channel or magma capacity.

estimated from the induction equation

$$
\operatorname{rot}\left(\boldsymbol{v} \times \boldsymbol{B}_{0}\right)=\Delta \boldsymbol{b} / \mu \sigma
$$

where $\sigma$ is the media conductivity, $v$ is the oscillations velocity, $\boldsymbol{B}_{0}$ is the Earth's magnetic field.

The magnetic field induced by the wave is approximately

$$
b_{z}=\mu \sigma B_{0 z} k_{x} v_{x} / k_{z}^{2}
$$

where $k_{x}$ and $k_{z}$ are horizontal and vertical components of the wave vector.

For inertial oscillations with $T=100 \mathrm{~s}$ according to (3.1) the induced magnetic field is $b_{z} \approx 0.1 \mathrm{nT}$, if $k_{x}=10^{-3} \mathrm{~m}^{-1}, v_{x}=f / k_{x}=10 \mathrm{~m} / \mathrm{s}$, $k_{z}=10^{-2} \mathrm{~m}^{-1}$. For inertial oscillations with $T=$ $=10-30 \mathrm{~s}$ the induced magnetic field is approximately $b_{z} \approx 0.01 \mathrm{nT}$ if $k_{x}=10^{-2} \mathrm{~m}^{-1}, v_{x}=$ $=1 /\left(f \cdot k_{x}\right)=6 \mathrm{~m} / \mathrm{s}, k_{z}=10^{-1} \mathrm{~m}^{-1}$. As the period of oscillation increases, the magnitude of the induced magnetic fields increases too.

\subsection{The magnetic fields induced by the vortex in intermediate volcano capacities}

A vortex in a conducting liquid in the presence of a constant magnetic field induces a 
constant azimuthal magnetic field (Parker, 1979)

$$
B_{\varphi}=\mu \sigma V_{\varphi} B_{0 z} D / 2
$$

where $V_{\varphi}$ is the rotation velocity, $D$ is the depth of rotating liquid.

The value of the magnetic field induced by magma movements in intermediate volcanic capacity is approximately $B_{\varphi}=2.5 \mathrm{nT}$ for $D=$ $=1000 \mathrm{~m}, v_{\varphi}=5 \mathrm{~m} / \mathrm{s}, s=10^{-1} \mathrm{Sm} \cdot \mathrm{m}^{-1}$.

The vertical magnetic field $b_{z}$ over the vortex is calculated as a field of circular current $j_{\varphi}=$ $=\sigma\left(\boldsymbol{v}_{r} \times \boldsymbol{B}_{0 z}\right)$

$$
b_{z}=\mu \iint_{S}(j / 2 r) d S
$$

where $S$ is the magma surface. Then the induced magnetic field is approximately $b_{z}=\mu \sigma \pi$. - $B_{0 z} R_{0} v_{z}$. The qualitative estimation gives a value $b_{z} \approx 10 \mathrm{nT}$ (see fig. 1 ).

The value of the magnetic field that can be measured on the Earth's surface, at distance $r$ from the magnetic field source is approximately

$$
b_{z}(r)=2 \mu \sigma \pi B_{0 z} R_{0}^{3} v_{z} / r^{2} .
$$

At distance $r=100 \mathrm{~km}$ from the volcanic center the measured magnetic field must be approximately $0.05-0.1 \mathrm{nT}$. The corresponding gradient of the magnetic field is approximately $\nabla b_{z} \approx 1 \mathrm{pT} / \mathrm{km}$.

These theoretical results are in accordance with the measurements. In practice, the vertical component of the magnetic fields decreases as $r^{-n}$ where $n \approx 1.2$ (Ismaguilov et al., 2001) and the gradient of the magnetic field is $1-5 \mathrm{pT} / \mathrm{km}$.

\section{Comparison with observed data}

Let us compare the described mechanism of magnetic field excitation with the results of actual measurements. Measurements of ULF electromagnetic disturbances were carried out in Japan before and during a period of seismic activity. (Ismaguilov et al., 2001). Behavior of the magnetic field prior to the earthquake has the following stages:

1) The magnitude of the magnetic field starts to increase $\approx 1.5$ months before the seismic activity in the frequency band 0.001-0.01 $\mathrm{Hz}$.

2) On the background of the common increase oscillations of the magnetic field value are observed with the period of approximately some days.

3) The magnetic field decreases sharply (1.5-2 days) prior to the earthquake.

According to the mechanism described above, the increase in the magnetic field just prior to an earthquake is related to the lifting of magma to the Earth's surface. Oscillations of the magnetic field can be related to changes in magma levels in volcanic channels and capacities and with inertial waves at the surface of magma.

Sharp decrease of the magnetic field 1.5-2 days before the earthquake can be explained by slowing of all movements in magma before the beginning of the earthquake, by the great amount of gas bubbles and/or hard particles that accumulate at the magma surface and difficulties in upwelling of magma and also vortex and wave movements in it.

The observed values of the magnetic fields are about $0.01-0.4 \mathrm{nT}$, that corresponds to the estimations made.

\section{Conclusions}

We can assume that hydrodynamic processes in magma are one of the possible causes of electromagnetic emission. In this work, three different possible mechanisms for excitation of magnetic fields were examined. These are:

i) magma flow in volcano channels;

ii) inertial waves at the magma surface that contribute to the magnetic field at the frequencies $0.01-0.1 \mathrm{~Hz}$;

iii) vortex flows that appear in magma when magma fills intermediate capacities.

A general increase in seismic activity leads to an intensification of the hydrodynamic processes in magma and to changes in electromagnetic emission. 


\section{Acknowledgements}

The authors are very grateful to Dr. RichertBoe for her help and useful discussions.

\section{REFERENCES}

Fedotov, S.A., I.S. UtKIN and L.I. UtKINA (2000): The estimation of scales of core volcano centers, Volcanol. Seismol., 3, 3-14.

ISMAGUilov, V.S., Yu. A. KopytenKo, K. Hattory, P.M. Voronov, O.A. Molchanov and M. Hayakawa (2001): ULF magnetic emissions connected with under sea bottom earthquakes, Natural Hazards Earth Sys. Sci., 1, 23-31.
MARChinIn, J.K. (1985): Volcanism (Nedra, Moscow), pp. 285 (in Russian).

MogI, K. (1985): Earthquake Prediction (Academic Press. Tokyo), pp. 355

PARKER, E.N. (1979): Cosmical Magnetic Fields. Their Origin and Their Activity (Claredon Press, Oxford), pp. 858.

Proussevitch, A.A., D.L. Sahagian and V.A. Kutolin (1993): Stability of foams in silicate melts, J. Volcanol. Geotherm. Res., 59 (1-2), 161-178.

SLEZIN, Yu.B. (1999): The Mechanism of Volcanic Eruption (a Stationary Model), p. 127 (in Russian).

StaceY, F.D. (1964): The seismomagnetic effect, Pure Appl. Geophys., 58, 5-22.

VAN'YAN, L.L. and P.P. SHILOVSKII (1983): The Deep Electroconductivity of Oceans and Continents (Nauka, Moscow), (in Russian). 